\title{
Alteraciones urinarias en niños litiásicos paraguayos según estado nutricional
}

\author{
Patricia Funes, Liliana Sosa, Valentina Díaz, Dominich Granado, Gloria Echagüe, \\ *Rosa Guillén \\ Universidad Nacional de Asunción, Instituto de Investigaciones en Ciencias de la Salud, Departamento de \\ Bioquímica Clínica, San Lorenzo, Paraguay
}

Cómo referenciar este artículo/ How to reference this article:
Funes P, Sosa L, Díaz V, Granado D, Echagüe G, Guillén R. Alteraciones urinarias en niños litiásicos paraguayos según estado nutricional. Mem. Inst. Investig. Cienc. Salud. 2019; 17(3): 28-33

\begin{abstract}
RES U M E N
La urolitiasis es una enfermedad multifactorial. En los últimos años se ha observado un incremento a nivel mundial de la incidencia de la litiasis urinaria tanto en adultos como en niños. Los cambios en los hábitos alimentarios y un aumento en la prevalencia de exceso de peso podrían asociarse a este fenómeno. El objetivo del trabajo fue describir la frecuencia de alteraciones urinarias presentes en niños con urolitiasis según estado nutricional. En este trabajo observacional transversal se incluyeron 104 niños litiásicos de ambos sexos divididos en dos grupos según estado nutricional: 68 niños con peso adecuado y 36 niños con sobrepeso u obesidad considerando criterios de la OMS. Se determinaron en orina de 24 horas calcio, fósforo, sodio, ácido úrico, citrato y magnesio. Del total de pacientes participantes 54 (51.9\%) fueron niñas y 50 (48,1\%) fueron niños. El 65,4\% de los niños presentó peso normal y el $34,6 \%$ de los niños sobrepeso u obesidad con edades medias de $10 \pm 4$ años y $8 \pm 4$ años respectivamente. El $80 \%$ de los niños presentó al menos una alteración urinaria, siendo las más frecuentes en ambos grupos la hipocitraturia e hiperuricosuria. Es llamativa la elevada frecuencia de alteraciones en las concentraciones de promotores e inhibidores de cristalización, en ambos grupos, indicando un riesgo aumentado de recidivas.
\end{abstract}

Palabras clave: Sobrepeso, urolitiasis, pediatría, urianálisis.

\section{Urinary alterations in Paraguayan lithiasic children according to nutritional status}

\begin{abstract}
A B S T R A C T
Urolithiasis is a multifactorial disease. Recently the incidence of urolithiasis in adults and children is increasing worldwide. Changes in eating habits and an increase in the prevalence of overweight could be associated with this phenomenon. The objective was to describe the frequency of urinary alterations according to nutritional status in children with urolithiasis. This cross-sectional observational study included 104 lithiasic boys and girls divided into two groups: 68 children with normal weight and 36 children with overweight or obesity considering WHO criteria. Calcium, phosphorus, sodium, uric acid, citrate and magnesium levels were determined in 24-hour urine. The frequency of boys and girls was $51.9 \%$ and $48.1 \%$ respectively. Sixty five percent of the children presented normal weight and $34.6 \%$ of the children were overweight or obese with mean ages of $10 \pm 4$ years and $8 \pm 4$ years respectively. Eighty percent of the children had at least one urinary disorder, the most frequent being hypocitraturia and hyperuricosuria in both groups. The high frequency of
\end{abstract}

- Los autores no declaran conflictos de intereses

Fecha de recepción: julio 2019. Fecha de aceptación: agosto 2019

*Autor correspondiente: Rosa María Guillén Fretes. Universidad Nacional de Asunción, Instituto de Investigaciones en Ciencias de la Salud. Número de teléfono: 595994318520

Email: rmguillenf@gmail.com 
alterations in the concentrations of urinary promoters and inhibitors of crystallization in both groups was remarkable, indicating an increased risk of recurrence.

Keywords: Overweight, urolithiasis, pediatrics, urinalysis.

\section{INTRODUCCIÓN}

La urolitiasis es una enfermedad multifactorial que se debe a un desequilibrio entre la cantidad de inhibidores y de promotores de la cristalización en la orina. Las diferentes alteraciones de la composición química de la orina pueden crear un medio favorable para la formación de cálculos renales. En los últimos años se ha producido un incremento de la incidencia de la litiasis urinaria tanto en adultos como en niños pudiendo asociarse a este fenómeno los cambios en los hábitos alimentarios y el exceso de peso. La incidencia de la litiasis renal en el niño no se conoce con exactitud, es variable en distintas regiones del mundo y resulta un problema de salud relevante debido a sus consecuencias sobre la funcionalidad renal ${ }^{(1-4)}$.

Diversos autores señalan que hasta un $30 \%$ de los niños litiásicos presentan un peso por encima del percentil 90. Sin embargo, existe controversia en los resultados de investigaciones en niños con urolitiasis y la influencia del estado nutricional. Algunos estudios señalan que se observan frecuencias superiores de hipocitraturia, hipercalcuria e hiperoxaluria en la población con exceso de peso y relacionan a la obesidad con mayor riesgo de aparición de cálculos renales ${ }^{(5-9)}$.

En este sentido el diagnóstico oportuno y la instauración de medidas correctivas o preventivas son fundamentales así como conocer las alteraciones urinarias presentes en los pacientes con esta patología.

En este contexto, este trabajo buscó describir la frecuencia de alteraciones urinarias presentes en niños paraguayos con urolitiasis de acuerdo al estado nutricional.

\section{MATERIALES Y MÉTODOS}

En este trabajo observacional de corte trasversal se incluyeron 104 niños de 3 a 17 años, con diagnóstico de urolitiasis confirmado por al menos un método de imagen (ecografía y/o tomografía), que no habían iniciado aún su tratamiento y que concurrieron al Instituto de Investigaciones en Ciencias de la Salud (IICS) entre los años 2010-2011. La población de estudio incluyó a pacientes que cumplieron los criterios de inclusión del protocolo de estudio aprobado por los Comités Científico y Ético del IICS (Código P17/2010). Fueron excluidos aquellos niños que recibían medicación específica para controlar trastornos metabólicos causantes de litiasis. Los padres o tutores de los participantes firmaron un consentimiento escrito.

Se registraron antecedentes personales y familiares de litiasis renal, presencia de otras enfermedades, la medicación, peso y talla para la evaluación del estado nutricional. La interpretación de los resultados de las mediciones antropométricas se realizó utilizando los indicadores de IMC para la edad o indicador peso/talla según corresponda considerando los criterios de la $\mathrm{OMS}^{(10)}$.

Se determinaron en orina de 24 horas: calcio, fósforo, magnesio, ácido úrico, citrato y sodio utilizando el autoanalizador químico CB 350i Wiener Lab (Roma, Italia), y el fotocolorímetro Biosystem BTS-310 (Barcelona, España) con kits comerciales (Wiener Lab, Argentina; LTA, Italia), las concentraciones de sodio en orina se determinaron con el equipo AVL 9120 (Georgia, Estados Unidos) por detección electrodo ión selectivo. Se consideraron como alteraciones urinarias: a) Hipercalciuria, cuando la medición de calcio urinario tuvo valores superiores a $4 \mathrm{mg} / \mathrm{kg} / 24 \mathrm{~h}$, b) Hiperuricosuria, cuando la medición de ácido úrico urinario fue superior a $10 \mathrm{mg} / \mathrm{kg} / 24 \mathrm{~h}$, c) Hiperfosfaturia, cuando el fosforo urinario fue superior a $25 \mathrm{mg} / \mathrm{kg} / 24 \mathrm{~h}$ d) hipomagnesiuria, cuando el magnesio urinario fue inferior a 1 $\mathrm{mg} / \mathrm{kg} / 24 \mathrm{~h}$, e) Hipocitraturia, cuando la excreción de citrato urinario tuvo valores inferiores a $8 \mathrm{mg} / \mathrm{kg} / 24 \mathrm{~h}$ e f) Hipernatruria cuando el sodio urinario superior niveles de 4,5 $\mathrm{mEq} / \mathrm{kg} / \mathrm{día}$. Se consideró diuresis disminuida a valores inferiores a $35 \mathrm{mg} / \mathrm{kg} / 24 \mathrm{hs}^{(11,12)}$. Además de detectar la frecuencia de alteraciones urinarias se realizaron mediciones séricas de calcio, magnesio, ácido úrico y fosforo.

El análisis de datos se realizó empleando el programa Epiinfo (versión 3.5.1, CDC, Atlanta). Se utilizaron la media y desvío estándar para expresar la excreción de los diferentes analitos en la población de estudio. Se utilizaron frecuencias para expresar las alteraciones metabólicas. Los test empleados fueron: $t$ student o Kruskal Wallis para 
comparación de medias o Chi cuadrado o Fischer para comparar las frecuencias entre los dos grupos de estudios con un nivel de significancia estadística de 0,05.

\section{RESULTADOS}

Del total de pacientes participantes $54(51.9 \%)$ eran niñas y $50(48,1 \%)$ eran niños. El grupo 1 incluyó a $68(65,4 \%)$ niños con peso normal y el grupo 2: aquellos con sobrepeso u obesidad representado por $36(34,6 \%)$ niños, y con edades medias de $10 \pm 4$ años y $8 \pm 4$ años respectivamente. La media de mediciones antropométricas y las diferencias estadísticas entre ambos grupos se observan en la Tabla 1.

Tabla 1: Características generales de los niños estudiados

\begin{tabular}{lcccc}
\hline Características & $\begin{array}{c}\text { Total } \\
(\mathbf{n = 1 0 4 )}\end{array}$ & $\begin{array}{c}\text { Grupo 1 (n=68) } \\
\text { Peso adecuado }\end{array}$ & $\begin{array}{c}\text { Grupo 2 (n=36) } \\
\text { Exceso de peso }\end{array}$ & P \\
\hline $\begin{array}{l}\text { Edad (años) } \\
\text { Sexo }\end{array}$ & $10 \pm 4$ años & $10 \pm 4$ años & $8 \pm 4$ años & $0.03^{* *}$ \\
\hline $\begin{array}{l}\text { Femenino } \\
\text { Masculino }\end{array}$ & $54(51.9 \%)$ & $38(55.9 \%)$ & $16(44,4 \%)$ & $0,13^{*}$ \\
$\begin{array}{l}\text { Mediciones } \\
\text { Antropométricas }\end{array}$ & $50(48,1 \%)$ & $30(44,1 \%)$ & $20(55,6 \%)$ & \\
$\begin{array}{l}\text { Peso (kg) } \\
\text { Talla (cm) }\end{array}$ & $38,0 \pm 16,0$ & $36,9 \pm 15,2$ & $40,1 \pm 17,5$ & $0,327^{* *}$ \\
IMC $\left(\mathbf{k g} / \mathbf{m}^{\mathbf{2}}\right)$ & $135,1 \pm 28,9$ & $140,3 \pm 22,6$ & $125,3 \pm 36,5$ & $0.054^{* *}$ \\
& $19,6 \pm 3,5^{\mathrm{a}}$ & $18,1 \pm 2,8^{\mathrm{b}}$ & $22,1 \pm 3,5^{\mathrm{c}}$ & $<0,001^{*}$
\end{tabular}

Nivel de significancia estadística $p \leq 0,05$. Test empleados para comparación de proporciones:* test de chi cuadrado. Test empleados para comparación de medias: **t student **Kruskal Wallis a) $n=92$ niños $\geq 5$ años, b) $n=61$ niños $\geq 5$ años, c) $n=31$ niños $\geq 5$ años.

Los antecedentes familiares de litiasis renal fueron del $50 \%$ en ambos grupos de niños. Se registraron recidivas de la enfermedad en $26(72,2 \%)$ de los niños con exceso de peso y en $34(50 \%)$ con peso adecuado $(p=0,04)$. La aparición de recidiva antes de los 5 años del primer episodio litiásico se observó en 37 niños (61,2\%).

El cólico renal y hematuria fueron las principales manifestaciones presentes en ambos grupos sin diferencia significativa. En menor frecuencia se observaron fiebre e infecciones urinarias a repetición.

La determinación de analitos séricos reveló hipercalcemia e hipomagnesemia en 10 $(9,6 \%)$ y $9(8,7 \%)$ niños respectivamente, en su mayoría normopesos. Se encontró un niño obeso con hiperuricemia.

La excreción promedio de sodio urinario fue mayor, mientras que la de citrato, magnesio y calcio fueron ligeramente menores en el grupo de pacientes con exceso de peso pero sin que estas diferencias sean significativas (Tabla 2). La diuresis inferior a $35 \mathrm{ml} / \mathrm{kg} / 24 \mathrm{hs}$ se encontró en el $48,7 \%$ y en el $63,9 \%$ de los niños con peso adecuado y exceso de peso, respectivamente.

Tabla 2: Valores de concentración de analitos urinarios

\begin{tabular}{|c|c|c|c|c|}
\hline & $\begin{array}{c}\text { Total }^{a} \\
(n=104)\end{array}$ & $\begin{array}{l}\text { Peso adecuado } \\
\quad(n=68)\end{array}$ & $\begin{array}{c}\text { Exceso de peso }^{a} \\
(n=36)\end{array}$ & $\mathbf{P}$ \\
\hline Citrato $(\mathrm{mg} / \mathrm{kg} / \mathrm{dia})$ & $8,0 \pm 5,5$ & $8,4 \pm 5,3$ & $7,3 \pm 5,8$ & $0,36^{*}$ \\
\hline Calcio (mg/kg/dia) & $2,8 \pm 2,1$ & $2,9 \pm 2,1$ & $2,5 \pm 1,9$ & $0,36 *$ \\
\hline $\begin{array}{l}\text { Ácido úrico } \\
(\mathrm{mg} / \mathrm{kg} / \mathrm{dia})\end{array}$ & $9,2 \pm 5,7$ & $9,2 \pm 6,2$ & $9,3 \pm 4,9$ & $0,93 *$ \\
\hline Sodio (meq/kg/dia) & $3,1 \pm 1,5$ & $3,0 \pm 1,3$ & $3,3 \pm, 1,6$ & $0,31 *$ \\
\hline Magnesio (mg/kg/dia) & $2,5 \pm 1,7$ & $2,5 \pm 1,7$ & $2,3 \pm 1,6$ & $0,58^{*}$ \\
\hline
\end{tabular}

a Media \pm desviación estándar. Nivel de significancia estadística $p \leq 0,05$. Test empleados para comparación de medias: *t student.

Se observó una o más alteraciones urinarias en el $82,4 \%$ y el $75 \%$ de los niños con peso adecuado y exceso de peso, respectivamente. En ambos grupos la presencia de una alteración única fue la situación más frecuente, llegando a cifra del $42,6 \%$ y $50 \%$ en niños 
con peso adecuado y con exceso de peso, respectivamente (Ver Figura 1). Así mismo en ambos grupos, cerca del $20 \%$ no presentó alteraciones urinarias en el momento de realizar el estudio metabólico. Las alteraciones urinarias más frecuentes fueron la hipocitraturia e hiperuricosuria en ambos grupos. La hipercalciuria estuvo presente en el $22 \%$ de los niños con peso adecuado y en el $11 \%$ de los niños con exceso de peso. La frecuencia de las mismas diferenciada por población se observa en la Tabla 3.

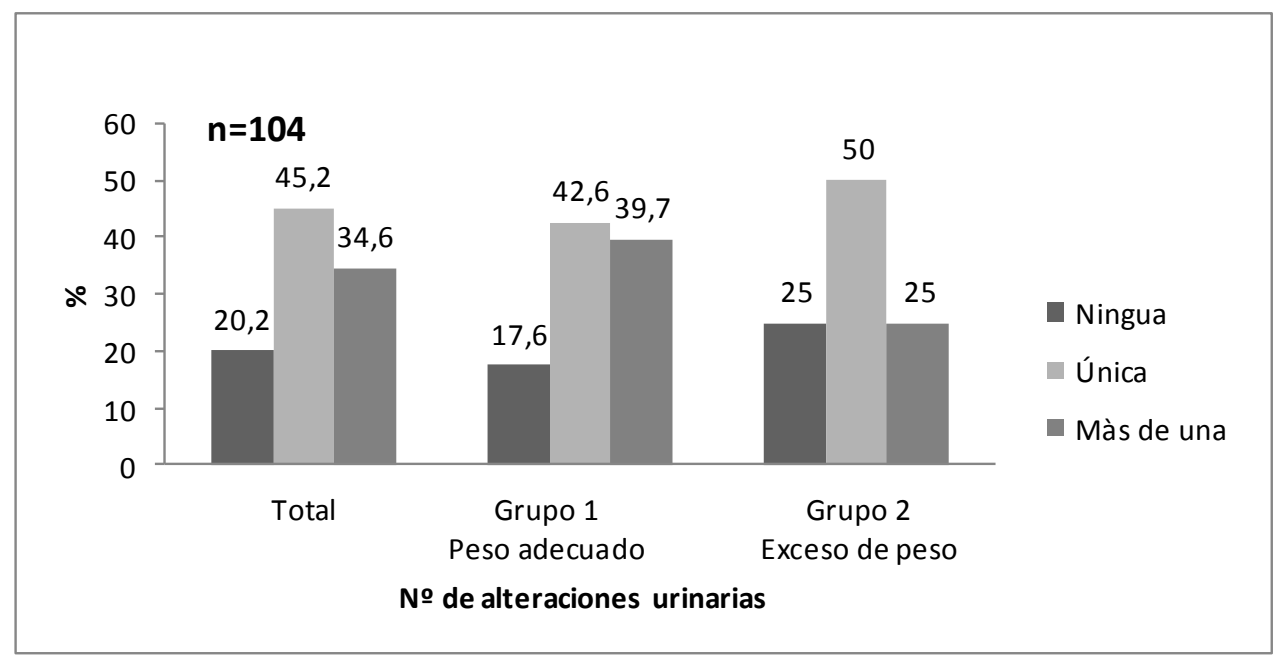

Figura 1: Frecuencia de alteraciones urinarias Frecuencia de alteraciones urinarias presentes en la población en general y diferenciada según estado nutricional

Tabla 3: Frecuencia de alteraciones urinarias

\begin{tabular}{lcccc}
\hline Alteraciones urinarias & $\begin{array}{c}\text { Total } \\
(\mathbf{n = 1 0 4 )}\end{array}$ & $\begin{array}{c}\text { Peso adecuado } \\
(\mathbf{n = 6 8 )}\end{array}$ & $\begin{array}{c}\text { Exceso de peso } \\
(\mathbf{n = 3 6 )}\end{array}$ & P \\
\hline Hipocitraturia $^{\mathrm{a}}$ & $52(50 \%)$ & $32(47,1 \%)$ & $20(55,6 \%)$ & NS* \\
Hipercalciuria $^{\mathrm{b}}$ & $19(18,3 \%)$ & $15(22,1 \%)$ & $4(11,1 \%)$ & NS** \\
Hiperuricosuria $^{\mathrm{c}}$ & $34(32,7 \%)$ & $24(35,3 \%)$ & $10(27,8 \%)$ & NS* \\
Hipernatruria $^{\mathbf{d}}$ & $13(12,5 \%)$ & $8(11,8)$ & $5(13.9 \%)$ & NS* \\
Hipomagnesuria $^{\mathrm{e}}$ & $12(11,5 \%)$ & $10(14,7 \%)$ & $2(5,6 \%)$ & NS** \\
\hline
\end{tabular}

a) Hipocitraturia: citrato urinario inferior a $8 \mathrm{mg} / \mathrm{kg} / 24 \mathrm{~h}$ b) Hipercalciuria: calcio urinario superior a $4 \mathrm{mg} / \mathrm{kg} / 24 \mathrm{~h} \mathrm{c)} \mathrm{Hiperuricosuria:} \mathrm{ácido} \mathrm{úrico} \mathrm{urinario} \mathrm{superior} \mathrm{a} 10 \mathrm{mg} / \mathrm{kg} / 24 \mathrm{~h}$ d) Hipernatruria cuando el sodio urinario superior niveles de 4,5 mEq/ $\mathrm{kg} / \mathrm{día}$ e) Hipomagnesiuria: magnesio urinario inferior a $1 \mathrm{mg} / \mathrm{kg} / 24 \mathrm{~h}$. NS: diferencia estadística no significativa. Nivel de significancia estadística $p \leq 0,05$. Test empleados para comparación de proporciones:* test de chi -cuadrado, **test exacto de Fischer.

\section{DISCUSIÓN}

En la población escolar y adolescente el aumento de la prevalencia de obesidad infantil se ha convertido en un problema de salud pública y nutrición. El porcentaje de obesidad y sobrepeso encontrado en la población estudiada es mayor a lo reportado a nivel nacional en niños no litiásicos. Según datos publicados en la Estrategia Nacional para la prevención y control de la obesidad 2015-2025 en escolares y adolescentes la cifra de sobrepeso y obesidad actualmente se aproxima al $30 \%$ En los niños y adolescentes el problema se hace más grave debido a que en ausencia de medidas correctivas a tiempo sobre sus hábitos, el niño obeso podría llegar a ser un adulto obeso y además asociarse a enfermedades tales como la hipertensión arterial, hiperinsulinemia, dislipidemia, diabetes mellitus tipo 2, entre otras $^{(13)}$. Estudios acerca de la relación entre el estado nutricional y alteraciones metabólicas en urolitiasis en niños han arrojado resultados controvertidos, atribuyendo además a otros factores el aumento de incidencia de la misma ${ }^{(14-16)}$.

La edad de inicio de la litiasis infantil es variable y generalmente se da entre los 5 y 15 $a_{n ̃ o s}{ }^{(17)}$. En este grupo de pacientes estudiados se observó esa tendencia de presentación en relación a la edad, a pesar de que en el grupo de pacientes con exceso de peso se dio a edades más tempranas. En relación al sexo, la frecuencia de presentación fue similar coincidiendo con lo reportado por estudios en niños litiásicos en Estados Unidos ${ }^{(7,18)}$. 
Al analizar las medias de concentraciones urinarias de los analitos litogénicos observamos que los valores de aquellos promotores como ácido úrico y sodio resultaron ligeramente superiores en aquellos con exceso de peso. Así también las concentraciones urinarias de los inhibidores tales como citrato y magnesio se encontraron ligeramente disminuidas en estos pacientes. Si bien estos resultados no presentaron diferencias estadísticamente significativas podrían señalar una tendencia hacia situaciones de riesgo litogénico caracterizados por aumento de concentración de promotores y disminución de inhibidores de cristalización en los niños con exceso de peso. De forma a aumentar el tamaño de la muestra estudiada y verificar si la tendencia observada se confirma o se descarta se continúa realizando el estudio de perfiles bioquímicos en niños con urolitiasis.

En ambos grupos, el perfil metabólico de la mayoría de los niños reveló una única alteración de parámetros urinarios apuntando hacia posibles procesos litogénicos monofactoriales. Este comportamiento difiere al de adultos, en quienes generalmente se presentan dos a más alteraciones en forma simultánea ${ }^{(17)}$. Con respecto a la presentación de las alteraciones urinarias, la hipocitraturia fue la más frecuente en ambos grupos pero no se asoció con el estado nutricional. Estos resultados son similares a estudios en niños de Corea $^{(19)}$, y con lo publicado en adultos a nivel nacional ${ }^{(20,21)}$, sin embargo, difieren con lo reportado por Bandari et al. en Estados Unidos en el 2016 quienes reportaron disminución del magnesio y citrato y aumento de la concentración del calcio urinario en el grupo de niños litiásicos con exceso de peso donde encontraron diferencia significativa en las concentraciones de los analitos urinarios ${ }^{(6)}$. Esta disminución en la concentración de citrato urinario podría estar relacionada al elevado consumo de proteínas animales y sodio, característico de los hábitos alimenticios de la población paraguaya. A esto se suma la diuresis insuficiente presente en ambos grupos y el clima caluroso característico del país durante la mayor parte del año que contribuyen a la aparición de litiasis urinaria.

En lo que respecta a factores de riesgo intrínsecos, la historia familiar de litiasis se presentó en la mitad de los niños en coincidencia con un estudio realizado en España donde el antecedente de litiasis familiar se dio en el $41,5 \%$ de los $\operatorname{casos}^{(22)}$ y a otro en Turquía donde reportaron antecedentes de urolitiasis en el $55,4 \%{ }^{(8)}$. La recidiva encontrada en ambos grupos fue elevada, y fue significativamente mayor en el grupo de pacientes con exceso de peso llegando a cifras superiores al $70 \%$. La patología litiásica se caracteriza por altas tasas de recidivas debido a la persistencia y variabilidad de factores de riesgo para cristalización, por tanto, el seguimiento apropiado es fundamental para corregir las alteraciones. En este sentido métodos sencillos como la cristaluria o índices de excreción en orina al azar permiten evaluar la eficiencia de medidas terapéuticas evitando los sesgos de las dificultades asociadas a la colecta de orina de 24 hs necesarias para estudio metabólico. Estos métodos se han implementado recientemente en el país y por tanto su uso apropiado ayudará en el seguimiento de los pacientes disminuyendo el riesgo de recurrencia.

El elevado riesgo de recurrencia es considerado un problema mayor de salud en pediatría y la frecuencia de la misma es equivalente a la de adultos. Existen estudios que reportan que el $50 \%$ de niños sufren recidivas 7 años posteriores al episodio litiásico ${ }^{(1,23)}$ y son similares a lo encontrado en los niños en este estudio. Las manifestaciones clínicas en los niños son variadas y no se centran en el cólico clásico de aparición frecuente en los adultos $^{(1)}$. En esta población si bien se registraron cólicos renales en un poco más de la mitad de los niños, la hematuria resultó otro síntoma que condujo a consulta médica y con ello al diagnóstico.

Una limitación de nuestro trabajo es la falta de acceso a datos clínicos que revelen la existencia de alteraciones anatómicas que contribuyan tanto al proceso litiásico y de recidiva. Los resultados de este trabajo señalan la alta tasa de alteraciones metabólicas únicas o combinadas revelando procesos metabólicos activos con riesgo de recidivas en la población de estudio. Se observó una tendencia de aumento de concentración de promotores y disminución de concentración de inhibidores en niños con exceso de peso, sin embargo, esas diferencias no fueron estadísticamente significativas. Estos resultados podrían estar indicando que el exceso de peso si bien no es la causa primaria podría ser un factor predisponente en la aparición de litiasis y sobretodo observado en edades más tempranas, pudiendo además deberse al tamaño de muestra por lo que se requiere de un estudio más amplio que permita observar estas variables en un mayor número de pacientes. Cabe destacar también que el exceso de peso en población pediátrica es preocupante no sólo por el riesgo de urolitiasis sino además por el riesgo de otras 
enfermedades crónicas que pueden disminuir la calidad de vida en la edad adulta, situación que requiere de un mayor énfasis a programas de educación que favorezcan hábitos saludables.

\section{REFERENCIAS BIBLIOGRAFICAS}

1. Penido MGMG, Tavares $M$ de $\mathbf{S}$. Pediatric primary urolithiasis: Symptoms, medical management and prevention strategies. World J Nephrol. 2015; 4(4):444-54.

2. Negri AL, Spivacow $F R$, Del Valle $E E$, Forrester M, Rosende G, Pinduli I. Role of overweight and obesity on the urinary excretion of promoters and inhibitors of stone formation in stone formers. Urol Res. 2008;36(6):303-7.

3. Kirejczyk JK, Korzeniecka-Kozerska A, Baran M, Porowska $H$, Porowski T, Wasilewska A. Dyslipidaemia in overweight children and adolescents is associated with an increased risk of kidney stones. Acta Paediatr. 2015;104(9):e407-13.

4. Areses Trapote, R. Enfermedad renal litiásica en la edad pediátrica. Evaluación diagnóstica y estudio metabólico. Pediatr Contin. 2012;10(5):243-56.

5. Eisner $\mathrm{BH}$, Eisenberg $\mathrm{ML}$, Stoller ML. Influence of body mass index on quantitative 24-hour urine chemistry studies in children with nephrolithiasis. J Urol. 2009;182(3):1142-5.

6. Bandari J, Dangle PP, Lyon TD, Lee A, Schneck FX, Cannon GM, et al. 24-Hour Urinary Parameters in Overweight and Obese Children with Urolithiasis. J Urol. 2016;196(2):526-30.

7. Sas DJ, Becton LJ, Tutman J, Lindsay LA, Wahlquist AH. Clinical, demographic, and laboratory characteristics of children with nephrolithiasis. 2016;44(3):241-6.

8. Yılmaz AÇ, Büyükkaragöz B, Oguz U, Çelik $B$. Influence of body mass index on pediatric urolithiasis. J Pediatr Urol. 2015;11(6):350.e1-350.e6.

9. Knoll T. Epidemiology, pathogenesis, and pathophysiology of urolithiasis. Eur Urol Suppl. 2010;9(12):802-6

10. Paraguay. Ministerio de Salud Pública y Bienestar Social. Instituto Nacional de Alimentación y Nutrición. Manual Básico de Evaluación Nutricional Antropométrica. Asunción: MSPBS; 2013

11. Areses Trapote R, Urbieta Garagorri MA, Ubetagoyena Arrieta M, Mingo Monge T, Arruebarrena Lizarraga D. Evaluación de la enfermedad renal litiásica. Estudio metabólico. Pediatr Barc. 2004; 61: 41827.

12. Caffarati Sfulcini J, Pérez-Carral Jr, Cosentino M, Bujons Tur A, Carat Barredo Jm, Villavicencio Mavrich H. Actualización en el manejo de la litiasis pediátrica. Rev Esp Pediatr. 2012;68(4):295-301.

13. Paraguay. Ministerio de Salud Pública y Bienestar Social. Estrategia Nacional para la Prevencion y el Control de la Obesidad 2015-2025 (Internet). Dirección de Vigilancia de Enfermedades No transmisibles. (citado 28 de agosto de 2019). Disponible en: http://portal.mspbs.gov.py/dvent/estrategi a-nacional-la-prevencion-control-laobesidad-2015-2025

14. Batavia JPV, Tasian GE. Clinical effectiveness in the diagnosis and acute management of pediatric nephrolithiasis. Int J Surg. 2016;36:698-704.

15. Miah T, Kamat D. Pediatric Nephrolithiasis: A Review. Pediatr Ann. 2017;46(6):e2424.

16. Kovesdy CP, Furth SL, Zoccali C; World Kidney Day Steering Committee. Obesity and kidney disease: hidden consequences of the epidemic. Kidney Int. 2017;91(2):260-262

17. Clayton DB, Pope JC. The increasing pediatric stone disease problem. Ther Adv Urol. 2011;3 (1):3-12.

18. Kim SS, Luan X, Canning DA, Landis JR, Keren R. Association between Body Mass Index and Urolithiasis in Children. J Urol. 2011;186(4 Suppl):1734-9.

19. Chung JD, Kim T-H, Myung SC, Moon YT, Kim KD, Chang IH. Influence of overweight on 24-hour urine chemistry studies and recurrent urolithiasis in children. Korean J Urol. 2012;53 (4):268-74.

20. Guillén R, Ruiz I, Stanley J, Ramirez A, Pistilli N. Evaluación de parámetros litogénicos en pacientes con urolitiasis que concurrieron al Instituto de Investigaciones en Ciencias de la Salud en el año 2009. Mem Inst Investig Cien Salud. 2010;8 (1): 14-21.

21. Funes $P$, Echagüe $G$, Ruiz I, Rivas $L$, Zenteno J, Guillén R. Lithogenic risk in patients from Paraguay with urolithiasis. Rev Med Chil. 2016;144(6):716-22.

22. Santo-Ruiz M, Hidalgo-Barquero E, GarcíaBlanco J. Urolitiasis en la infancia: revisión clínica y epidemiológica de los últimos años en nuestro medio. Vox Paediatrica. 2004;12(1):13-19.

23. Gajengi AKR, Wagaskar VG, Tanwar HV, Mhaske S, Patwardhan SK. Metabolic Evaluation in Paediatric Urolithiasis: A 4Year Open Prospective Study. J Clin Diagn Res JCDR. 2016;10(2): PC04-6. 